\title{
Direction of Arrival Estimation Using Augmentation of Coprime Arrays
}

\author{
Tehseen Ul Hassan * (1), Fei Gao, Babur Jalal and Sheeraz Arif \\ School of Information and Electronics, Beijing Institute of Technology, Beijing 100081, China; \\ gaofei@bit.edu.cn (F.G.); Baburjalal@hotmail.com (B.J.); sheeraz.arif@bit.edu.cn (S.A.) \\ * Correspondence: tehseen@bit.edu.cn; Tel.: +86-010-6891-7546
}

Received: 17 September 2018; Accepted: 6 November 2018; Published: 9 November 2018

\begin{abstract}
Recently, direction of arrival (DOA) estimation premised on the sparse arrays interpolation approaches, such as co-prime arrays (CPA) and nested array, have attained extensive attention because of the effectiveness and capability of providing higher degrees of freedom (DOFs). The co-prime array interpolation approach can detect $\mathrm{O}(\mathrm{MN})$ paths with $\mathrm{O}(\mathrm{M}+\mathrm{N})$ sensors in the array. However, the presence of missing elements (holes) in the difference coarray has limited the number of DOFs. To implement co-prime coarray on subspace based DOA estimation algorithm namely multiple signal classification (MUSIC), a reshaping operation followed by the spatial smoothing technique have been presented in the literature. In this paper, an active coarray interpolation (ACI) is proposed to efficiently recovering the covariance matrix of the augmented coarray from the original covariance matrix of source signals with no vectorizing and spatial smoothing operation; thus, the computational complexity reduces significantly. Moreover, the numerical simulations of the proposed ACI approach offers better performance compared to its counterparts.
\end{abstract}

Keywords: coarray interpolation; degree of freedom; nuclear norm minimization; DOA estimation; co-prime array; MUSIC; uniform linear array; sparse array

\section{Introduction}

Direction of arrival estimation (DOA) is a vast research topic due to its broad range of applications in many areas such as sonar, radar and wireless communication. It is widely known that traditional high resolution DOA estimation algorithms like estimation of signal parameters via rotational invariance techniques (ESPRIT) and multiple signal classification (MUSIC) [1,2], which can only resolve $N$ sources with uniform linear array (ULA) of $N+1$ sensors, hence it has $\mathrm{N}$ degree of freedom (DOF). It requires a larger number of antenna elements to resolve more number of sources, which would acquire enormous cost but still can't get remarkable DOA estimation efficiency and accuracy.

To address these challenges and resolve more sources than the actual physical sensors, numerous array geometry and signal processing techniques have been presented [3,4]. Recently, minimum redundancy arrays (MRAs) [3], which can enhance the number of contiguous sensors with a certain number of physical antenna elements. However, MRA has no general expression. Additionally, MRA estimates that the proficient covariance matrix conforming to a broad antenna array needs a relatively intricate and tedious iterative operation. To overcome this problem, an approach named as the nested arrays has been presented in [4]. In the nested array, nonuniform arrays designed by integrating multiple ULAs where every sub-array has a distinct inter-element spacing. It is a fact that some array elements are placed very closely in the nested array; therefore, mutual coupling effects among the sensors can turn into compelling form and thus compromise the effective design of sparse array and the performance. Since there is no approximate DOF and no closed-form expression for the 
array geometry in MRA and the existence of mutual coupling problems between contiguous antenna elements in the nested array design, it is difficult to construct the system model in most of the cases.

Most recently, co-prime arrays (CPA) [5-7] sparked the research activities and received considerable attention by taking closed-form expressions into account for coarray geometry and contraction of the mutual coupling effect in the direction of arrival estimation problem. The simplicity and high efficiency of CPA make it more attractive among the sparse interpolation approaches. With the utilization of a co-prime array approach, the powers of signal behave as new coherent sources [1]. CPA consists of two subarrays with $M$ and $N$ elements, where $M$ and $N$ are co-prime integers. The co-prime array interpolation approach can detect $\mathrm{O}(\mathrm{MN})$ sources with $\mathrm{O}(\mathrm{M}+\mathrm{N})$ antenna elements. The number of DOFs is an essential coarray design benchmark because high number of DOFs means more paths can be detected by the antenna array. However, the corresponding difference coarray set in the sparse array has holes or missing elements that cause some limitations in DOA estimation. Subsequently, the DOFs are limited for subspace based algorithms, like ESPRIT and MUSIC, since spatial smoothing based operations are generally applicative to only uniform arrays [8-10]; therefore, an augmented array is constructed from the co-prime array to obtain uniformity in the array to estimate DOA.

Several array augmentation approaches have been designed to address this problem. In [11], the authors used array motion to brim the missing elements in the corresponding difference coarray set; however, this approach enforces some limitations on the motion status of the array and received data at a precise point. In [12], the author utilized the covariance matrix augmentation approach, but it required complex matrix completion operation to wholly exploit the offered DOFs. In [8], the author used a co-prime sampling technique based on spatial smoothing, in order to construct a positive semidefinite matrix from the covariance matrix corresponding to the received data vector. The optimal positive semidefinite matrix can be directly utilized on a subspace based algorithm such as MUSIC. To resolve the holes case in the difference coarray set [13], the author proposed a sparse signal reconstruction approach by vectorizing the original covariance matrix; therefore, the number of detectable paths is reduced to the positive lags. In [14], central contagious array elements are used to estimate DOA and ignore the holes in the difference array set. The author suggests using the central contagious ULA to perform DOA estimation, although the DOF is not fully exploited, but the complexity of the system reduces to a significant level. The thinned co-prime array structure in [15], introduced to exploit the redundancy in the corresponding difference coarray. The contribution of a few sensors in the subarray 2M-sensor of the conventional co-prime can be gathered by the rest of the sensors in the array; therefore, these elements are removed without upsetting the properties of the actual array, hence more uniform lags and aperture with a low number of sensors. In [16], a low complex compressive sensing approach has been proposed. The author combined the auto-correlation and cross-correlation matrices of both subarrays to cope with the redundant entries and complexity without sacrifising the DOA estimation performance. Nuclear norm minimization approach in [17] has been utilized to brim the holes in the difference coarray set. An augmented array with a larger aperture is constructed from the sparse array by reshaping the original covariance matrix corresponding to a received data vector. Then, utilizing the spatial smoothing operation to recovering a rank of matrix; thereafter, it is used for DOA estimation.

In this paper, we proposed an efficient approach named as an active coarray interpolation (ACI) to obtain rigorous DOA estimation with low complexity. The covariance matrix corresponding to the coarray is constructed from the original dispersion matrix (covariance matrix) of the received source data with no vectorizing and spatial smoothing operation, which reduces the computational complexity with respect to the existing approaches; in the final step, the MUSIC algorithm is applied to find the DOA. Moreover, the proposed approach effectively exploited the co-prime array to fill the holes in the corresponding difference coarray, thus increasing the number of degrees of freedom (DOFs) for the subspace based MUSIC algorithm. The numerical simulation is carried out to demonstrate the efficiency of the proposed ACI approach. 
The rest of this paper is arranged into the following sections. System Model and different coarray interpolations approaches have been discussed in Sections 2 and 3, respectively, whereas, in Section 4, the proposed active interpolation approach has been presented. We analyze the performance of proposed and existing approaches by conducting numerical simulation in Section 5. Finally, in Section 6, we draw the conclusions.

Notations: Scalars represented by (a) lower-case letters, vectors represented by $(\boldsymbol{a})$ lower-case bold letters, matrices denoted by $(A)$ upper-case letters in bold, and sets are expressed by $\mathbb{A}$ upper-case letters in blackboard boldface. Particularly, $I_{M}$ stands for the identity matrix of $M \times M$ dimension. Similarly, $A^{*}$ denoted the conjugate, the transpose is represented by $A^{T}$ and the complex conjugate transpose of $A$ is represented by $A^{H}$. The Kronecker product represented $\otimes, v e c(\cdot)$ stands for the vectorization operation of a matrix, and $\operatorname{diag}(A)$ stands for the diagonal elements of matrix $A$. $|\mathbb{A}|$ refers to the cardinality of the $\mathbb{A} . \operatorname{tr}(A)$ denoted the trace of matrix $A$. The nuclear norm and the Frobenius norm of matrix $A$ is represented by $\|A\|_{*}$ and $\|A\|_{F}$, respectively. $E[\cdot]$ represented the expectation operator.

\section{System Model}

The co-prime array that is produced by a set of co-prime integers $(M, N)$ shows in Figure 1. With no loss of generality, we consider that the subarray $M$ has less sensors than that of $\mathrm{N}(M<N)$. The zeroth position of the both subarrays shares the same sensor [8,18-22]. $M+N-1$ comprises all of the elements in the corresponding CPA. The first coarray has $2 M$ having $N_{d}$ inter element spacing, whereas the second array has $N$ sensors having $M_{d}$ inter-element spacing. In the co-prime arrays, the corresponding integer set $(\mathbb{S})$ is expressed as

$$
\mathbb{S}=\left\{M_{n}, 0 \leq n \leq N-1\right\} \bigcup\left\{N_{m}, 0 \leq m \leq 2 M-1\right\} .
$$

The physical location of antenna elements are $\mathbb{L}=\left\{b_{1}, b_{2}, \ldots, b_{L}\right\} \times d_{0}=\mathbb{S} \times d_{0}$, where $\mathbb{S}$ is the corresponding integer set. $d_{0}=\lambda / 2$ is the distance between antenna elements, whereas $\lambda$ is known as wavelength. The corresponding difference set $(\mathbb{D})$ is used to exploit the DOF offered by co-prime design is given as

$$
\mathbb{D}=\left\{c_{1}-c_{2} \mid c_{1} \in \mathbb{S}, c_{2} \in \mathbb{S}\right\} .
$$

Moreover, the locations of elements in the corresponding difference set $(\mathbb{D})$ are given as $\mathbb{D} \times d_{0}$. The corresponding difference set $(\mathbb{D})$ is a non-uniform array; therefore, the existence of holes in the corresponding difference set limits the maximum DOF and it can't be directly utilized on DOA estimation algorithms. To make it hole free array, we take $\mathbb{S}$ as the physical location of the sensors and its corresponding difference coarray is $(\mathbb{D})$; then, the maximum central contiguous ULA $(\mathbb{U})$ in the corresponding difference coarray $(\mathbb{D})$ is given as

$$
\mathbb{U}=\{m \mid\{m|, \ldots,| m \mid \mathbb{S}\} \subseteq \mathbb{S}\} .
$$

The maximum central contiguous ULA $(\mathbb{U})$ of the corresponding difference coarray $(\mathbb{D})$ has less sensors, which reduces the DOFs. To achieve a full degree of freedom and implement that array on a subspace based MUSIC algorithm, it is required to exploit the difference coarray to get a maximum number of DOFs and uniform array. By taking these considerations into account, the augmented ULA $(\mathbb{V})$ constructed that has range from $-(2 M-1) N$ to $(2 M-1) N$, expressed as

$$
\mathbb{V}=\{m \mid\{\min (\mathbb{D}) \leq m \leq \max (\mathbb{D})\} .
$$

The cardinalities defined above for co-prime arrays are given as

$$
\begin{array}{ll}
|\mathbb{S}|=2 M+N-1 & |\mathbb{U}|=2 M N+2 M-1, \\
|\mathbb{D}|=3 M N+M-N & |\mathbb{V}|=4 M N-2 N+1 .
\end{array}
$$




\section{Subarray 1}

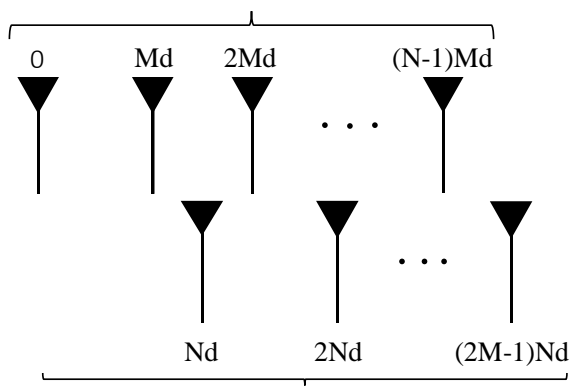

Subarray 2

Figure 1. Coprime array geometry.

Assume that $K$ narrow band and uncorrelated signals with powers $\left[\sigma_{1}^{2}, \sigma_{2}^{2}, \ldots, \sigma_{K}^{2}\right]$ impinging on the array, embedded in Additive white Gaussian noise (AWGN) from angle $\left\{\theta_{1}, \theta_{2}, \ldots, \theta_{K}\right\}$. The received signals at time " $t$ " which is gathered by all the antenna elements are given as

$$
x(t)=A s_{k}(t)+n(t) \quad t=1,2,3, \ldots, T,
$$

where

$$
A=\left[a_{S}\left(\theta_{1}\right), a_{S}\left(\theta_{2}\right), \ldots, a_{S}\left(\theta_{K}\right)\right]
$$

is the steering vector of dimension $(2 M+N-1)$ towards the direction $\theta_{K} . \quad s_{k}(t)=$ $\left[s_{1}(t), s_{2}(t), \ldots, s_{K}(t)\right]^{T}$ is the Kth signal from the source. The number of snapshots is denoted as " $T$ ", and $n(t)$ is the independent and uncorrelated noise vector having the length $(2 M+N-1)$. The steering vector $A$ corresponding towards the direction $\theta_{K}$ is given as

$$
a_{\mathbb{S}}\left(\theta_{K}\right)=\left[e^{j 2 p d_{1} d_{0} \sin \left(\theta_{1}\right) / \lambda}, \ldots, e^{j 2 p d_{L} d_{0} \sin \left(\theta_{K}\right) / \lambda}\right]^{T} .
$$

Theoretically, the covariance matrix of data vector $x(t)$ can be given as

$$
R_{\mathbb{S}}=E\left[x(t) x^{H}(t)\right]=A R_{s S} A^{H}+\sigma_{n}^{2} I_{L},
$$

where $E\{\cdot\}$ represented the statistical expectation operator and $R_{s s}=\operatorname{diag}\left[\sigma_{1}^{2}, \sigma_{2}^{2}, \ldots, \sigma_{K}^{2}\right]$, which is also equal to $E\left[s(t) s^{H}(t)\right]$ is the covariance matrix corresponding to the source data with $\sigma_{k}^{2}$ signifying the power of the Kth source. $\sigma_{n}^{2}$ is the noise variance, and $I$ is an identity matrix of dimension $(2 M+N-1) \times(2 M+N-1)$. Consider that $R_{\mathbb{S}}$ is unattainable in practice but the maximum likelihood $(M L)$ method can be used to estimate $\tilde{R}_{\mathbb{S}}$ using the available samples as

$$
\tilde{R}_{\mathbb{S}}=\sum_{t=1}^{T} x_{\mathbb{S}}(t) x_{\mathbb{S}}^{H}(t)
$$

The entries of the covariance matrix $R_{\mathbb{S}}$ correspond to different lags. By vectorizing the covariance matrix, we obtain the autocorrelation vector $x_{\mathbb{D}}$.

$$
x_{\mathbb{D}}=\operatorname{vec}\left\{R_{\mathbb{S}}\right\}=\bar{A} p+\sigma_{n}^{2} \bar{I}=\left(A^{*} \odot A\right) p+\sigma_{n}^{2} \bar{I},
$$

where the vectorization operator is denoted as $\operatorname{vec}(\cdot)$, which converts a given matrix into a column vector. $\bar{A}$ is the array manifold matrix of dimension $(2 M+N-1)^{2} \times 1$, corresponding to the difference coarray, the source power is $p=\left[\sigma_{1}^{2}, \sigma_{2}^{2}, \ldots, \sigma_{K}^{2}\right]$, whereas $\odot$ and $(\cdot)^{*}$ respectively represent the Khatri-Rao product and the complex conjugation operator. $\bar{I}$ is the vectorized identity matrix. 


\section{Coarray Interpolation}

\subsection{MUSIC Algorithm Based on Central Contiguous ULA}

In the vector $\bar{x}_{\mathbb{D}}$ in (10), we see the coherent source like behavior " $\mathrm{p}$ ", whereas $\sigma_{n}^{2} \bar{I}$ as a deterministic noise term. However, there is only single snapshot available. Therefore, this case has similarities with coherent sources. Moreover, the holes exist in the difference coarray set $(\mathbb{D})$ as mentioned in Section 2; therefore, it can't be directly implemented in DOA estimation algorithms. To implement in a MUSIC algorithm, which is a subspace based high resolution algorithm to estimate DOA of the incoming signal, spatial smoothing is used to restore the rank of the signal covariance matrix; therefore, the consecutive lags mainly around the origin of difference co-array set are taken into account. The central contagious ULA range from $-l_{\zeta}$ to $l_{\zeta}$, where $-l_{\zeta}$ is minimum value and $l_{\zeta}$ is the maximum value in the consecutive lags range $[14,17,23-25]$. By removing the duplicated rows in (10) and sorting the rest of the rows from $-l_{\zeta}$ to $l_{\zeta}$, we obtain

$$
x_{\mathbb{U}}=\tilde{A} p+\sigma_{n}^{2} \tilde{I}
$$

The length of $x_{\mathbb{U}}$ is the same as the number of unique elements in the central uniform array of $2 l_{\zeta}+1$. $\tilde{A}$ behaves like an array manifold matrix corresponding to the central uniform array. $\tilde{I}$ is a $\left(2 l_{\zeta}+1\right) \times 1$ vector whose $\left(l_{\zeta}+1\right)$ th elements are 1 and the rest are zeros.

$x_{\mathbb{U}}$ represents a data vector on the central ULA $\mathbb{U}$. Then, this virtual array divide into $l_{\zeta}+1$ overlapping subarrays $\left\{X_{\mathbb{U} 1}, \ldots, X_{\mathbb{U}(\zeta+1)}\right\}$, where $X_{\mathbb{U} i}$ with the array elements positioned at $-i+1+k$ with $i=1,2, \ldots ., l_{\zeta}+1$ having $k=0,1, \ldots ., l_{\zeta}$. Then, we get

$$
R_{\mathbb{U}}=\frac{1}{l_{\zeta}+1} \sum_{i=1}^{l_{\zeta}+1} \tilde{x}_{\mathbb{U} i} \tilde{x}_{\mathbb{U} i}^{H}
$$

The optimal solution of (12), is teoplitz matrix, so that the MUSIC algorithm can be directly implemented to estimate DOA of incoming signal.

\subsection{Hybrid Coarray Interpolation}

In Hybrid coarray interpolation [23], the missing lags filled by coarray interpolation to exploit maximum number of DOFs, which is followed by a denoising operation. The base line for denoising operation is the existence of an error $E$ as a result of finite snapshots in (9), which is a general case. Therefore, $\tilde{R}_{\mathbb{S}}$ is given as

$$
\tilde{R}_{\mathbb{S}}=A R_{S S} A^{H}+E .
$$

$E$ contains $\sigma_{n}^{2} I$, the power matrix of noise. Conducting a series of multiple operations such as reshaping and coarray interpolation, the error stemming from finite snapshots, $E$, still exists in another shape, which is represented by $\tilde{E}$. Then, $\tilde{R}_{\mathbb{V}}$ can be expressed as

$$
\tilde{R}_{\mathbb{V}}=R_{\mathbb{V}}+\tilde{E}
$$

Instead of using spatial smoothing covariance matrix to suppress such kind of error, the hybrid interpolation approach utilizes the covariance matrix of coarray by exploiting the Hermitian Teoplitz structure, which can be shown as follows:

$$
R_{\mathbb{V}}=T(u)=\left[\begin{array}{cccc}
u_{1} & u_{2} & \ldots & u_{\left|V^{+}\right|} \\
u_{2}^{H} & u_{1} & \ldots & u_{\left|V^{+}\right|-1} \\
\vdots & \vdots & \ddots & \vdots \\
u_{\left|V^{+}\right|} & u_{\left|V^{+}\right|-1} & \ldots & u_{1}
\end{array}\right]
$$


Then, to eliminate the error as a result of the finite snapshot, an optimized denoising operation is designed by using the nuclear norm minimization, which is expressed as

$$
\begin{aligned}
& \min \|T(u)\|_{*} \\
& \text { subject to }\left\|\tilde{R}_{\mathbb{V}}-T(u)\right\|_{F} \leq \in,
\end{aligned}
$$

where the $\in$ depends on the noise variance.

\section{Active Coarray Interpolation (ACI)}

The structure of the corresponding difference set $(\mathbb{D})$ is non-uniform, and it has holes or missing lags so it can not be directly utilized to estimate DOA on the subspace based MUSIC Algorithm because it is unable to produce true DOA estimates, when numerous incoming signals hit on the array. To resolve this case, central contiguous ULA interpolation of the corresponding difference set $(\mathbb{D})$ presented in [6], but the maximum number of DOFs can not be exploited by using this method. On the other hand, array augmentation is used to interpolate holes [6,17]; therefore, we get a new augmented uniform linear array $\mathbb{V}$ with a larger aperture, it can be defined as $\mathbb{V}=\{m \mid \min (\mathbb{D}) \leq m \leq \max (\mathbb{D})\}[6,8,17]$. The retrieval of missing correlation information in covariance matrix of coarray $\left(R_{\mathbb{V}}\right)$ can be defined as a nuclear norm minimization case. The objective function is the convex case in nuclear norm minimization can be resolved by a software package CVX. In the hybrid coarray interpolation [23], the missing lags filled by coarray interpolation to exploit maximum number of DOFs, which is followed by a denoising operation. However, these methods required a series of operations such as reshaping, spatial smoothing operation and denoising measures to recover the covariance matrix of coarray from the corresponding original covariance matrix of received data, which increases the computational complexity.

In this paper, we proposed an efficient approach named as active coarray interpolation (ACI) to obtain rigorous DOA estimation with low complexity. The covariance matrix corresponding to the coarray is actively constructed from the original dispersion matrix (covariance matrix) of the received source data with no vectorizing and spatial smoothing operation, which reduces the computational complexity. The recovery of $R_{\mathbb{V}}$ from the original covariance matrix of received data $R_{\mathbb{S}}$ can be given as

$$
\begin{gathered}
R_{\mathbb{V}}=\arg \min \left\|\tilde{R}_{\mathbb{S}}\right\|_{*} \\
\text { subject to } R_{\mathbb{V}}=\tilde{R}_{\mathbb{S}}^{H}, \\
\left\langle R_{\mathbb{V}}\right\rangle_{c_{1}, c_{2}}=\left\langle R_{\mathbb{S}}\right\rangle_{c_{1}-c_{2}}
\end{gathered}
$$

By utilizing nuclear norm minimization, the number of resolvable sources are equal to $L=(|\mathbb{V}|+1) / 2=(4 M N-2 N+2) / 2$, it is equal to the number of positive lags in $\mathbb{V}$. The optimal solution of (17) contains Hermitian Teoplitz matrix $\left(R_{\mathbb{V}}\right)$, so it can be directly employed in estimating the coarray MUSIC spectra.

By conducting eigen decomposition of the covariance matrix $R_{\mathbb{V}}$ of coarray, we can obtain the noise subspaces $\left(U_{N}\right)$ and signal subspaces $\left(U_{S}\right)$, which can be expressed as

$$
\tilde{\theta}=\left[\begin{array}{ll}
U_{S} & U_{N}
\end{array}\right] \sum\left[\begin{array}{l}
U_{S}^{H} \\
U_{N}^{H}
\end{array}\right]
$$

It is known that the noise subspaces contain eigenvectors corresponding to the lowest eigenvalues and also $U_{S}$ is orthogonal to the $U_{N}$. Each row of steering vector is corresponding to the direction of a signal source, and it concludes that the source steering vectors are perpendicular to the $U_{N}$,

$$
a^{H}(\theta) U_{N}=0
$$


The reciprocal of the matrix product $\left(a^{H}(\theta) U_{N} U_{N}^{H} a(\theta)\right)$ produces largest peaks at the angle of arrival. Thus, spatial MUSIC spectra is defined as

$$
P_{M U S I C}=\frac{1}{a^{H}(\theta) U_{N} U_{N}^{H} a(\theta)} .
$$

Comparison to the existing approaches, the proposed active interpolation approach (ACI) have the following advantages:

1. The existing approaches recover the covariance matrix of the augmented coarray from the original covariance matrix of a source signal needs reshaping and spatial smoothing operations, while the $\mathrm{ACI}$ approach does not need these operations; consequently, the intricacy of the proposed approach reduced significantly.

2. MUSIC Algorithm Based on Central Contiguous ULA only focus on the consecutive lags mainly around the origin of difference co-array set, while the proposed ACI approach utilizes the augmented coarray set $(\mathbb{V})$; hence, it achieves a higher number of DOF.

3. Experimentally compared results with counterparts specifies that the proposed ACI offers better performance in terms of complexity, Root Mean Square Error (RMSE) and probability of resolution. The outlines of ACI approach is presented in the Table 1.

Table 1. Active Coarray Interpolation (ACI) approach.

\begin{tabular}{ll}
\hline Input & The received signals \\
Output & Direction of arrival estimation \\
\hline Step 1 & Construct Covariance Matrix $R_{\mathbb{S}}$ \\
Step 2 & Optimize $R_{\mathbb{S}}$ to get Toeplitz matrix $R_{\mathbb{V}}$ from (17) \\
Step 3 & Obtain noise subspace $U_{N}$ by conducting eigen-decomposition of $R_{\mathbb{V}}$. \\
Step 4 & Compute (20) and to get sharp peaks correspond to the direction of the sources. \\
\hline
\end{tabular}

\section{Simulation Results and Discussion}

In this section, we examined the performance of the proposed approach by conducting a series of simulations. The computational time calculated in MATLAB using the CVX package under the same environment of Intel CPU i3-3240 with a clock speed of $3.40 \mathrm{GHz}$ and 4 GB RAM. The experiment was conducted for 30 runs. The contiguous Based ULA approach takes 66.8127 seconds, the hybrid approach takes 72.3951 seconds and the proposed ACI takes $57.9326 \mathrm{~s}(13.29 \%$ less than a Contiguous ULA based approach and $19.97 \%$ less than the hybrid approach). Though the computational reduction is significant, but still the computational burden caused by the eigen decomposition.

In the following subsections, we presented the cardinalities of the co-prime array with respect to DOFs. Then, we compare the spatial spectrum of the proposed and existing approaches listed in this paper. The performance of the proposed approach in terms of Root Mean Square Error (RMSE) probability of Resolution against the signal-to-noise ratio (SNR) and snapshots by conducting Monte Carlo experiments. Moreover, to study the practical implications, we conducted a series of experiments under different scenarios to test the angular resolution capabilities in the last subsection.

\subsection{DOF Comparison}

Figure 2 depicts the co-prime Array configuration. The lags are represented by the cross $(*)$ and holes by the circles (०). The number of DOF is an essential coarray design benchmark and it is determined by the cardinalities of array with respect to $R_{\mathbb{S}}, R_{\mathbb{D}}, R_{\mathbb{U}}$ and $R_{\mathbb{V}}$. The DOFs increased with increasing number of lags in the array $[17,26]$. We consider a co-prime integer pair $(M=3$, $N=5$ ) for an extended co-prime array. The array consists of 10 sensors with the physical locations at $\mathbb{S}=\{0,2,5,6,9,10, \ldots, 25\}$. Similarly, the corresponding difference coarray $(\mathbb{D})$ is non uniform, and it 
has holes while it extends from -25 to 25 , i.e., $\mathbb{D}=\{0, \pm 1, \ldots, \pm 17, \pm 19, \pm 20, \pm 22, \pm 25\}$. The central contiguous is $\mathbb{U}=\{0, \pm 1, \pm 2, \ldots, \pm 17\}$ and the augmented array with no holes extends from -25 to 25 , i.e., $\mathbb{V}=\{0, \pm 1, \pm 2, \ldots, \pm 25\}$.

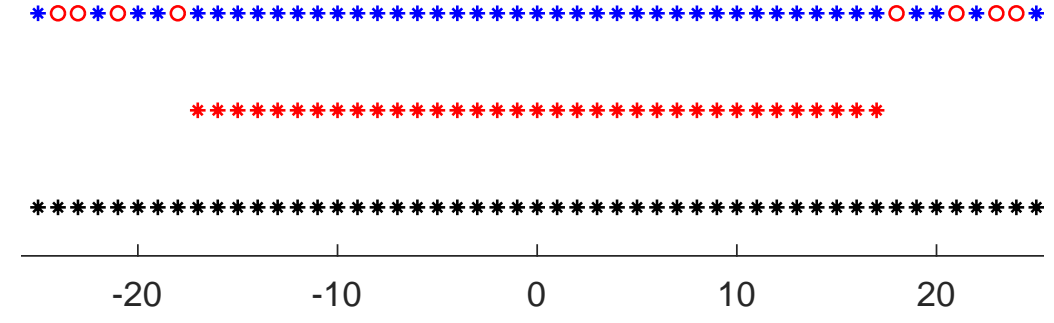

Figure 2. CPA Configuration ( $\circ$ Holes $*$ lags) (from the top) sensors in the corresponding CPA; the corresponding difference coarray; central contagious ULA; augmented coprime array.

\subsection{MUSIC Spectrum}

The spatial spectrum of the proposed approach is compared with the interpolated coarrays described in Section 3. In this simulation, we consider 16 sources (uncorrelated) with SNR of $0 \mathrm{~dB}$ and 500 snapshots. Figure 3 presents the normalized MUSIC spectrum of the uniformly distributed between -0.5 to 0.5 .

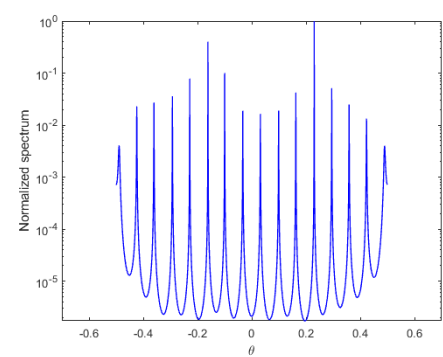

(a)

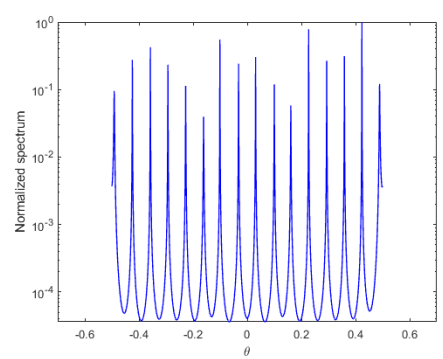

(b)

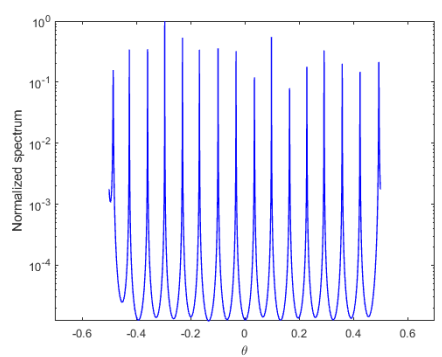

(c)

Figure 3. Spatial MUSIC spectrum (a) central ULA Based Approach; (b) hybrid Approach; (c) active coarray interpolation approach.

\subsection{Root Mean Square Error (RMSE)}

We examined the root mean square error (RMSE) $[6,27,28]$ performance in this section, against SNR and snapshots with $K=16$ uncorrelated sources. We conducted $I=300$ independent Monte Carlo experiments. The average RMSE of estimated DOAs is given as

$$
R M S E=\sqrt{\frac{\sum_{i=1}^{I} \sum_{k=1}^{K}\left(\tilde{\theta}_{k}(i)-\theta_{k}\right)^{2}}{I K}},
$$

where $\theta_{k}$ is the true angle and the estimated angles are denoted by $\tilde{\theta}_{k}(i)$.

Figures 4 and 5 depict the RMSE performance against SNR (varies from $-20 \mathrm{~dB}$ to $20 \mathrm{~dB}$ ) and snapshots (varies from 10 to 100) respectively. The performance of the hybrid approach is comparable to our proposed approach, but it is computationally more complex as described in the previous section. Figure 4 shows that the DOA estimation errors change (reduce) with respect to increasing SNR. Similarly, Figure 5 shows that DOA estimation error reduces with an increasing number of snapshots. As we can see, the proposed active interpolation approach performs better than its counterparts. 


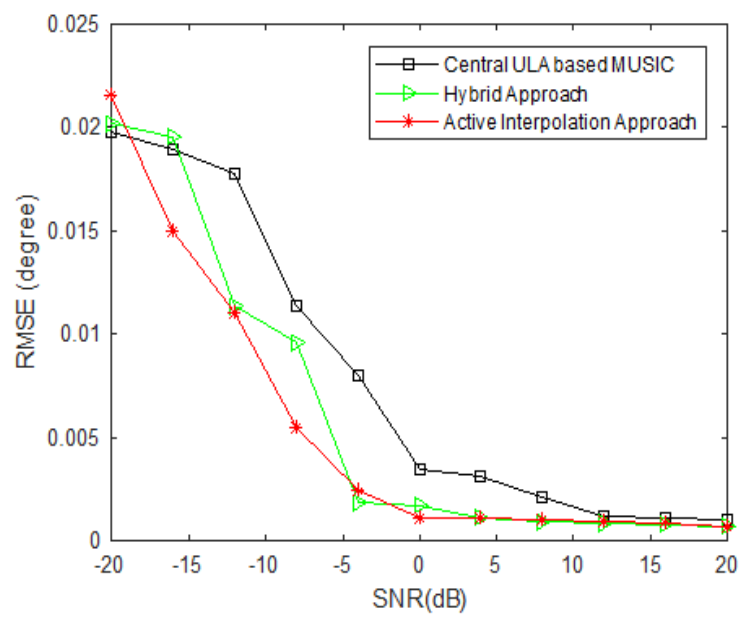

Figure 4. RMSE versus SNR (Snapshots $=100, K=16$ sources, $I=300$ Monte Carlo Experiments).

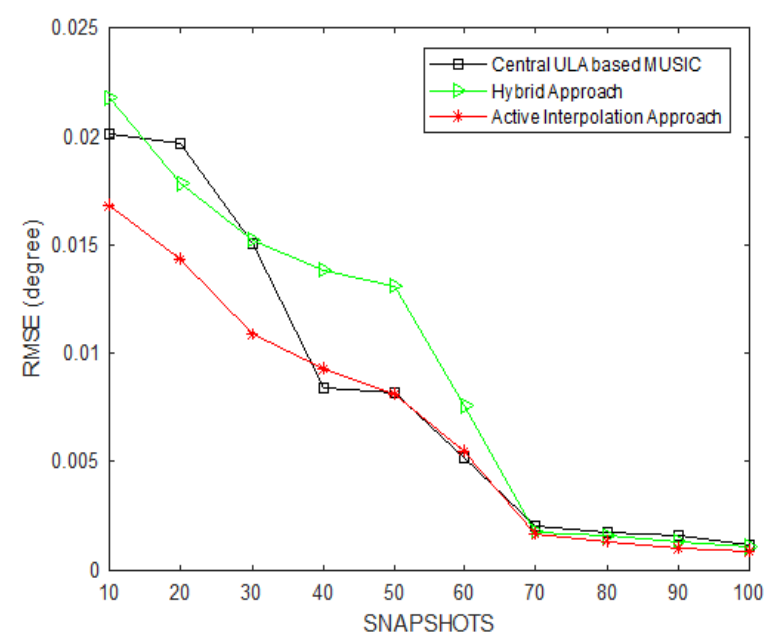

Figure 5. RMSE versus number of snapshots ( $\mathrm{SNR}=0 \mathrm{~dB}, K=16$ sources, $I=300$ Monte Carlo Experiments).

\subsection{Probability of Resolution}

We examined the performance in terms of Probability of Resolution (PR) $[29,30]$ of proposed approach in this section. The probability resolution is expressed as

$$
\text { Probabilty of Resolution }=\frac{P_{r}}{P}
$$

where $P$ is total number of experiments, and $P r$ is the number of successful results. The successful estimation, where the absolute direction of arrival estimation error, is within 1 degree.

Figures 6 and 7 show that the probability resolution against the SNR started from $-20 \mathrm{~dB}$ to $20 \mathrm{~dB}$ and snapshots (range from 10 to 100), whereas 16 coherent number of sources and the total number of trails are 300 . From the Figure 6, it can be noticed that, above $-10 \mathrm{~dB}$, all of the approaches obtain a $100 \%$ successful estimation. For every approach, the probability of successful estimation decreasing at a particular position is called the SNR threshold, unless it finally approaches a minimum level. The simulation results of a proposed active interpolation approach in Figures 6 and 7 outperform its counterparts. 


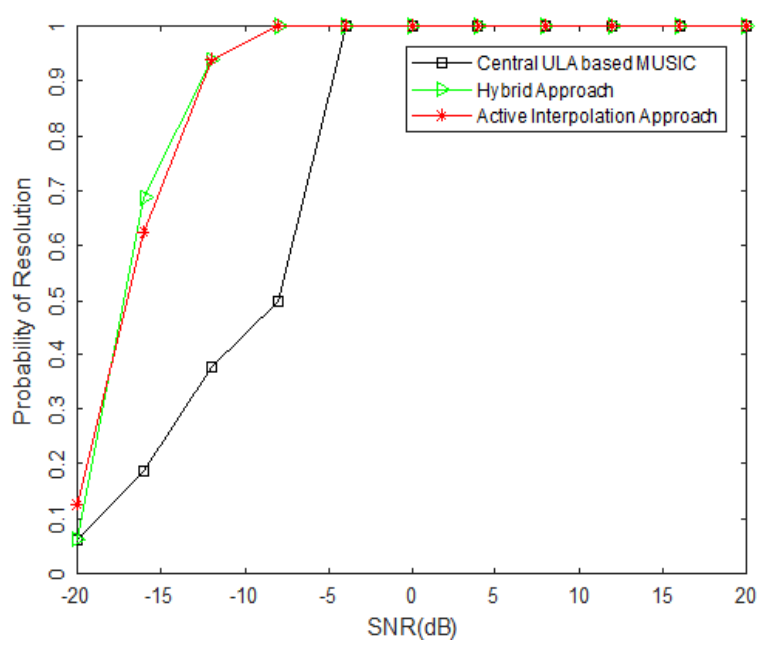

Figure 6. PR versus SNR (Snapshots $=100, K=16$ sources, $I=300$ Monte Carlo Experiments).

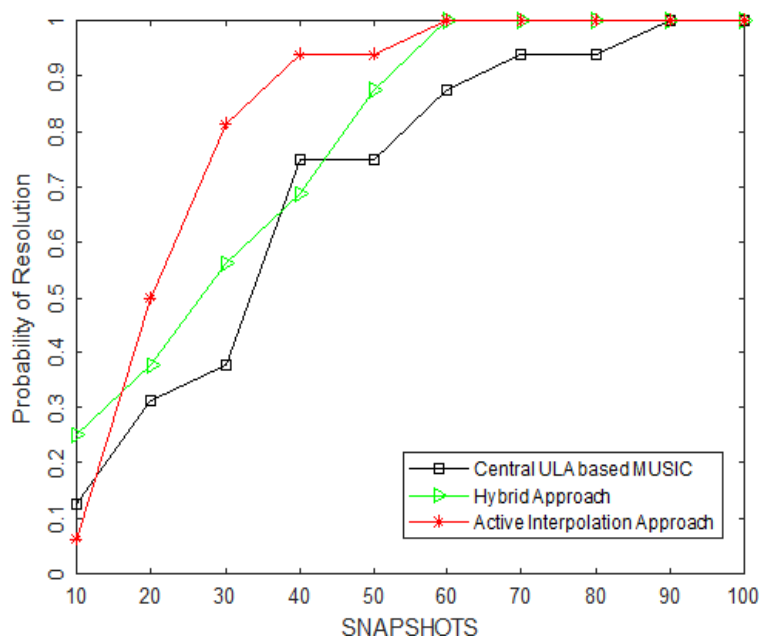

Figure 7. $\mathrm{PR}$ versus number of snapshots $(\mathrm{SNR}=0 \mathrm{~dB}, K=16$ sources, $I=300$ Monte Carlo Experiments).

\subsection{Angular Resolution}

We conducted numerical experiments under different scenarios to test the angular resolution capabilities. In the numerical simulation, the two close sources located at $\theta_{1}=0.10$ and $\theta_{2}=0.12$. The experiment was carried out with different values of SNR and snapshots for each scenario. We can see from Figure 8 that the contiguous ULA based approach and hybrid interpolation approach fail to identify the two close sources for low SNR and snapshots, while the proposed ACI resolves successfully.

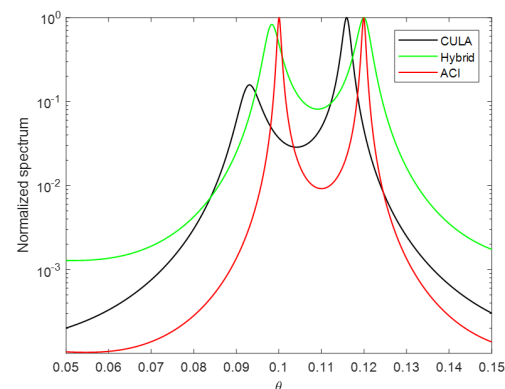

(a)

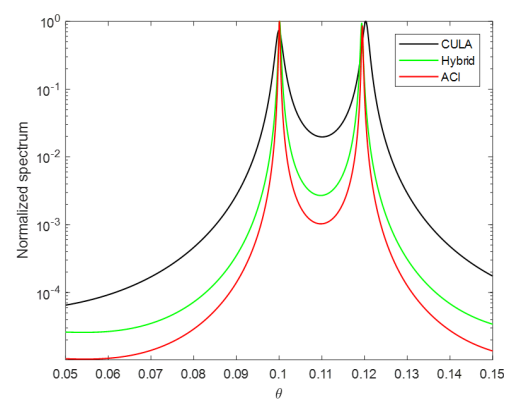

(b)

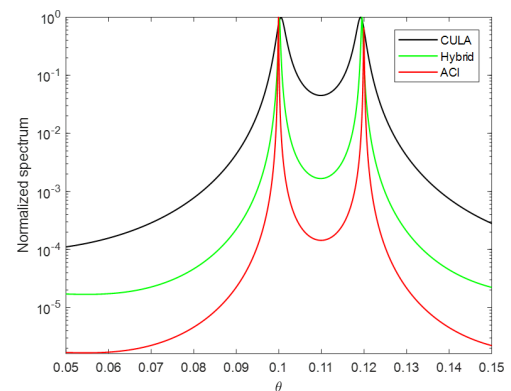

(c)

Figure 8. Angular Resolution of two close sources $\left(\theta_{1}=0.10\right.$ and $\left.\theta_{2}=0.12\right)$ with different scenarios (a) $\mathrm{SNR}=0 \mathrm{~dB}$, Snapshots = 100; (b) SNR = $5 \mathrm{~dB}$, Snapshots = 200; (c) SNR = $10 \mathrm{~dB}$, Snapshots $=300$. 


\section{Conclusions}

In this paper, we proposed an active coarray interpolation (ACI) approach based on co-prime array. The essential contribution of the proposed approach is the active construction of the covariance matrix of the augmented coarray from the original covariance matrix of source signal with no reshaping and spatial smoothing operation, which significantly reduces the computational complexity. Moreover, the proposed approach effectively exploited the coprime array interpolation to brim the holes in the corresponding difference coarray, thus increasing the number of degrees of freedom (DOFs) for the subspace based MUSIC algorithm. The simulation results have demonstrated that the proposed approach offers better performance in terms of Root Mean Square Error (RMSE), Probability of Resolution and the Angular Resolution compared to its counterparts.

Author Contributions: T.U.H. proposed the original idea, designed the experiments and wrote the paper under the supervision of F.G., B.J. and S.A. revised the manuscript. All authors read and approved the final manuscript.

Funding: This work was supported by the National Natural Science Foundation of China under Grant 61620106001.

Acknowledgments: The authors would like to thank all anonymous reviewers and editors for their helpful suggestions for the improvement of this paper.

Conflicts of Interest: The authors declare no conflict of interest.

\section{References}

1. Schmidt, R. Multiple emitter location and signal parameter estimation. IEEE Trans. Antennas Propag. 1986, 34, 276-280. [CrossRef]

2. Roy, R.; Kailath, T. ESPRIT-estimation of signal parameters via rotational invariance techniques. IEEE Trans. Acoust. Speech Signal Process. 1989, 37, 984-995. [CrossRef]

3. Moffet, A. Minimum-redundancy linear arrays. IEEE Trans. Antennas Propag. 1968, 16, 172-175. [CrossRef]

4. Liu, C.L.; Vaidyanathan, P.P. High order super nested arrays. In Proceedings of the IEEE Sensor Array and Multichannel Signal Processing Workshop (SAM), Rio de Janeiro, Brazil, 10-13 July 2016; pp. 1-5. [CrossRef]

5. Vaidyanathan, P.P.; Pal, P. Sparse Sensing With Co-Prime Samplers and Arrays. IEEE Trans. Signal Process. 2011, 59, 573-586. [CrossRef]

6. Qin, S.; Zhang, Y.D.; Amin, M.G. Generalized Coprime Array Configurations for Direction-of-Arrival Estimation. IEEE Trans. Signal Process. 2015, 63, 1377-1390. [CrossRef]

7. Liu, C.L.; Vaidyanathan, P.P. Remarks on the Spatial Smoothing Step in Coarray MUSIC. IEEE Signal Process. Lett. 2015, 22, 1438-1442. [CrossRef]

8. Pal, P.; Vaidyanathan, P.P. Coprime sampling and the music algorithm. In Proceedings of the Digital Signal Processing and Signal Processing Education Meeting (DSP/SPE), Sedona, AZ, USA, 4-7 January 2011; pp. 289-294. [CrossRef]

9. Shan, T.J.; Wax, M.; Kailath, T. On spatial smoothing for direction-of-arrival estimation of coherent signals. IEEE Trans. Acoust. Speech Signal Process. 1985, 33, 806-811. [CrossRef]

10. Pal, P.; Vaidyanathan, P.P. Nested Arrays: A Novel Approach to Array Processing With Enhanced Degrees of Freedom. IEEE Trans. Signal Process. 2010, 58, 4167-4181. [CrossRef]

11. Ramirez, J.; Krolik, J. Multiple source localization with moving co-prime arrays. In Proceedings of the IEEE International Conference on Acoustics, Speech and Signal Processing (ICASSP), Brisbane, QLD, Australia, 19-24 April 2015; pp. 2374-2378. [CrossRef]

12. Abramovich, Y.I.; Spencer, N.K.; Gorokhov, A.Y. Positive-definite Toeplitz completion in DOA estimation for nonuniform linear antenna arrays. II. Partially augmentable arrays. IEEE Trans. Signal Process. 1999, 47, 1502-1521. [CrossRef]

13. Zhang, Y.D.; Amin, M.G.; Himed, B. Sparsity-based DOA estimation using co-prime arrays. In Proceedings of the IEEE International Conference on Acoustics, Speech and Signal Processing, Vancouver, BC, Canada, 26-31 May 2013; pp. 3967-3971. [CrossRef]

14. Qin, S.; Zhang, Y.D.; Amin, M.G.; Himed, B. DOA estimation exploiting a uniform linear array with multiple co-prime frequencies. Signal Process. 2017, 130, 37-46. [CrossRef] 
15. Raza, A.; Liu, W.; Shen, Q. Thinned co-prime arrays for DOA estimation. In Proceedings of the 25th European Signal Processing Conference (EUSIPCO), Kos, Greece, 28 August-2 September 2017; pp. 395 -399. [CrossRef]

16. Liu, A.; Yang, Q.; Zhang, X.; Deng, W. Direction-of-Arrival Estimation for Coprime Array Using Compressive Sensing Based Array Interpolation. Int. J. Antennas Propag. 2017, 2017, 1-10. [CrossRef]

17. Liu, C.L.; Vaidyanathan, P.P.; Pal, P. Coprime coarray interpolation for DOA estimation via nuclear norm minimization. In Proceedings of the IEEE International Symposium on Circuits and Systems (ISCAS), Montréal, QC, Canada, 22-25 May 2016; pp. 2639-2642. [CrossRef]

18. Wu, C.; Zhang, M.; Wang, K. Underdetermined DOA estimation based on covariance matrix reconstruction using co-prime array. In Proceedings of the International Conference on Wireless Communications, Signal Processing and Networking (WiSPNET), Montreal, QC, Canada, 22-25 May 2017; pp. 671-674. [CrossRef]

19. Tan, Z.; Eldar, Y.C.; Nehorai, A. Direction of Arrival Estimation Using Co-Prime Arrays: A Super Resolution Viewpoint. IEEE Trans. Signal Process. 2014, 62, 5565-5576. [CrossRef]

20. Zhou, C.; Shi, Z.; Gu, Y.; Goodman, N.A. Doa estimation by covariance matrix sparse reconstruction of co-prime array. In Proceedings of the IEEE International Conference on Acoustics, Speech and Signal Processing (ICASSP), Brisbane, QLD, Australia, 19-24 April 2015; pp. 2369-2373. [CrossRef]

21. Wang, X.; Wang, X. DOA estimation with k-times extended co-prime arrays. In Proceedings of the 51st Asilomar Conference on Signals, Systems, and Computers, Pacific Grove, CA, USA, 29 October-1 November 2017; pp. 1183-1187. [CrossRef]

22. BouDaher, E.; Ahmad, F.; Amin, M.G. Sparsity-based extrapolation for direction-of-arrival estimation using co-prime arrays. In Proceedings of the SPIE, Baltimore, MD, USA, 17-21 April 2016; Volume 9857. [CrossRef]

23. Guo, M.; Chen, T.; Wang, B. An Improved DOA Estimation Approach Using Coarray Interpolation and Matrix Denoising. Sensors 2017, 17, 1140. [CrossRef] [PubMed]

24. Shi, Z.; Zhou, C.; Gu, Y.; Goodman, N.A.; Qu, F. Source Estimation Using Coprime Array: A Sparse Reconstruction Perspective. IEEE Sens. J. 2017, 17, 755-765. [CrossRef]

25. Shen, Q.; Liu, W.; Cui, W.; Wu, S.; Zhang, Y.D.; Amin, M.G. Low-Complexity Direction-of-Arrival Estimation Based on Wideband Co-Prime Arrays. IEEE/ACM Trans. Audio Speech Lang. Process. 2015, 23, 1445-1456. [CrossRef]

26. Yang, M.; Haimovich, A.M.; Yuan, X.; Sun, L.; Chen, B. A Unified Array Geometry Composed of Multiple Identical Subarrays With Hole-Free Difference Coarrays for Underdetermined DOA Estimation. IEEE Access 2018, 6, 14238-14254. [CrossRef]

27. Rao, B.D.; Hari, K.V.S. Performance analysis of Root-Music. IEEE Trans. Acoust. Speech Signal Process. 1989, 37, 1939-1949. [CrossRef]

28. Tan, Z.; Nehorai, A.; Eldar, Y.C. Continuous sparse recovery for direction of arrival estimation with co-prime arrays. In Proceedings of the IEEE 8th Sensor Array and Multichannel Signal Processing Workshop (SAM), A Coruna, Spain, 22-25 June 2014; pp. 393-396. [CrossRef]

29. Wang, Y.; Trinkle, M.; Ng, B.W.H. DOA Estimation under Unknown Mutual Coupling and Multipath with Improved Effective Array Aperture. Sensors 2015, 15, 30856-30869. [CrossRef] [PubMed]

30. Semira, H.; Belkacemi, H.; Marcos, S. High-Resolution Source Localization Algorithm Based on the Conjugate Gradient. EURASIP J. Adv. Signal Process. 2007, 2007, 073871. [CrossRef]

(C) 2018 by the authors. Licensee MDPI, Basel, Switzerland. This article is an open access article distributed under the terms and conditions of the Creative Commons Attribution (CC BY) license (http://creativecommons.org/licenses/by/4.0/). 